\title{
基于泊松分布和分形几何的甲骨拓片字形复原 方法
}

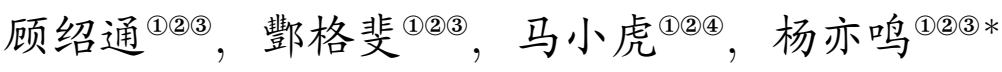 \\ (1) 徐州师范大学语言研究所, 徐州 221009 \\ (2) 江苏省语言科学与神经认知工程重点实验室, 徐州 221009 \\ (3) 徐州师范大学语言科学学院, 徐州 221009 \\ (4) 苏州大学计算机科学与技术学院, 苏州 215006 \\ * 通信作者. E-mail: yangym@xznu.edu.cn
}

收稿日期: 2009-11-02；接受日期: 2010-03-02 国家自然科学基金（批准号：30740040)、教育部人文社会科学基金（批准号：10YJC-740032)、江苏省社会科学基金（批准号： 09YYB011)、江苏高校哲学社会科学重点研究基地重大项目 “甲骨文的数字化处理及应用”、徐州师范大学社会科学基金 (批准号: 08XWA04) 和江苏省“333 高层次人才培养工程”基金 (批准号: 苏教办师 [2008]46 号) 资助项目

\begin{abstract}
摘要 文中提出了一种基于泊松分布和分形几何的甲骨拓片字形复原方法. 分析了甲骨拓片上字 形图像和噪声区域的分布特征, 通过计算每一个连通区域与拓片上所有连通区域数学期望的差值来 识别拓片上的噪声区域. 小于数学期望的连通区域被识别为噪声区域并被填充, 从而保留了字形笔 划区域. 分析了甲骨拓片上字形图像边缘的分形几何特征, 计算甲骨拓片字形边缘的分形维数等参 数, 通过计算拓片字形的轮廓线上当前点与左右相邻点形成的向量夹角的余弦值提取特征点, 并对 甲骨拓片字形边缘进行加权坐标的压缩变换，从而使甲骨拓片字形边缘得到平滑．实验结果显示， 该方法可以有效地去除甲骨拓片上的噪声区域, 保留字形笔划区域, 并使甲骨拓片上字形边缘得到 平滑.
\end{abstract}

关键词甲骨拓片 泊松分布 分形几何 分形维数 压缩变换 字形复原

\section{1 引言}

图像复原是计算机图形学和计算机视觉的一个研究热点, 在文物保护、视频特技、虚拟现实以及 消除多余物体等方面扮演着重要角色. 20 世纪以来, 随着考古工作的深入开展, 陆续出土了大量的古 代珍贵文献, 这些出土文献中有相当一部分是书写在龟甲和兽骨上面的, 如甲骨文 ${ }^{1)}$, 是我国迄今发 现的最早的一种成熟文字系统, 具有极其重要的学术价值和文化遗产保护价值. 甲骨文是书写在龟甲 和兽骨上的文字, 由于龟甲和兽骨质地本身并非平滑如镜, 又深埋地下经历了几千年的岁月沧桑, 加 上发掘和运输的污损, 损坏已很严重, 字形的边缘已经非常模糊, 这样受污损的甲骨文字形不利于在 数字图书馆和甲骨文字库建设中的进一步使用, 必须进行复原处理. 目前采用某些图像处理软件可以

1) 甲骨文是刻写在龟甲和兽骨上的中国古代文字系统, 流行于商朝 
近似恢复甲骨拓片字形的原始面貌, 但是涉及非常复杂的手工交互, 而且需要具有丰富经验的专业人 员操作, 非常耗时耗力, 而且修复效果也不如人意.

目前, 已出现多种图像修复算法, 如 Bertalmio 等 ${ }^{[1]}$ 提出了偏微分方程的图像复原方法, 但是这 种方法只适用于小尺度数字图像, 而且并不稳定. Chan 等 ${ }^{[2,3]}$ 提出了整体变分方法和基于曲率的扩 散模型方法, 这种方法依赖于边缘和边界曲率的连续性, 可以处理较大的区域, 但是处理后的边缘变 得非常模糊. Oliveira 等 [4] 提出了基于图像滤波的高斯卷积核的方法, 该方案可以快速恢复破损区域, 但是只适用于较小的破损区域. Carr ${ }^{[5]}$ 提出了径向基函数的修复方法, 该方案构造基于非均匀采样点 构造连续隐函数, 从而重建破损区域. Esedoglu 等 ${ }^{[6]}$ 提出一种基于 Mumford-Shah-Euler 图像模型的 修复方法, 该方案依赖于图像的先验模型, 将图像修复转换为计算一个泛函数的极值, 处理过程非常 复杂. Criminisi 等 ${ }^{[7]}$ 提出了纹理合成的方法, 可以处理大块破损区域, 然而效果并不如意. Elad 等 ${ }^{[8]}$ 提出了一种形态组件分析的图像修复方法. Bertozzi 等 ${ }^{[9]}$ 提出了基于 Cahn-Hilliard 方程的图像修复 方法. Guerrero-Coln 等 [10] 提出了一种空间变量高斯尺度混合的图像修复方法. Henri 等 ${ }^{[11]}$ 提出了 一种基于统计框架扩展学习的在线图像恢复方法, 但是该方案需要手工交互. Dobrosotskaya 等 ${ }^{[12]}$ 提 出了基于小波拉普拉斯变换的方法. 以上方法均采用图像处理的方法修复破损区域, 并没有分析噪声 区域的统计特征, 而且对于像甲骨拓片这种书写材料比较特殊、污染原因多样化以及埋藏时间漫长的 字形图像缺乏针对性, 修复效果也难以如意.

本文提出了基于泊松分布和分形几何的复原方法. 分析了甲骨拓片上字形图像和噪声区域的统计 分布特征, 对连通区域进行基于泊松分布的识别处理, 对噪声区域进行填充. 分析了甲骨拓片上字形 图像边缘的分形几何特征, 通过计算甲骨拓片字形图像边缘的分形维数, 对字形边缘进行加权的压缩 变换, 从而对字形边缘进行平滑.

\section{2 基于泊松分布的区域填充方法}

\section{1 甲骨拓片字形图像分析}

甲骨文是契刻在龟甲和兽骨上的文字, 因而字形的笔划线条具有以下特点: 构成甲骨文字形的线 条多为直线, 且线条瘦劲挺直, 两端尖锐, 转折处往往以两条直线相接成屈折状 [13]. 甲骨拓片在地下 深埋上千年, 由于受到腐蚀、发掘损坏以及拓片质地本身等原因, 导致甲骨拓片有许多噪声点, 如图 1, 2 所示. 图 3 是甲骨拓片字形图像的轮廓图. 甲骨拓片上的噪声点具有如下特点:

(1) 噪声区域的亮度低于甲骨拓片上字形笔划的亮度;

(2) 噪声区域呈离散状态, 连通区域面积较小, 区域面积服从泊松分布.

相比之下, 甲骨拓片上字形图像的特征有:

(1) 分形: 甲骨拓片在地下深埋上千年, 由于受到腐蚀, 甲骨拓片字形图像边缘部分与整体具有相 似性, 具备分形特征, 如图 3 所示;

(2) 笔划区域连通性: 甲骨拓片字形的笔划一般是单连通区域, 个别笔段会出现断裂;

(3) 像素值空间的聚玫性: 甲骨拓片字形笔划的像素亮度较高, 而且比较连续, 且在某个像素值区 域比较集中.

设随机变量 $X$ 服从泊松分布, 即 $X \sim P(\lambda)$, 可以表示如下:

$$
P_{i}=P\{X=i\}=\frac{\lambda^{i}}{i !} \mathrm{e}^{-\lambda} \quad(i=0,1,2, \ldots),
$$




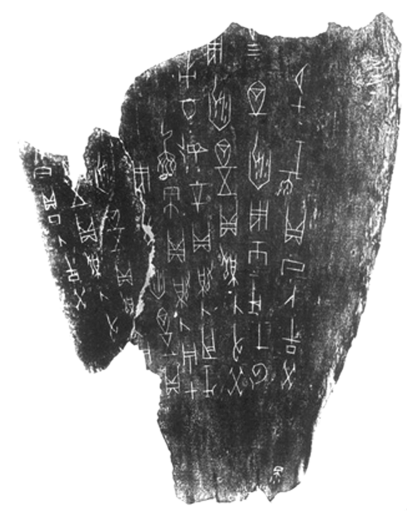

图 1 《甲骨文合集》559(正面)

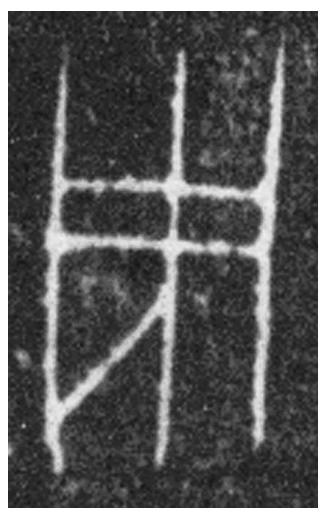

图 2 甲骨拓片字形

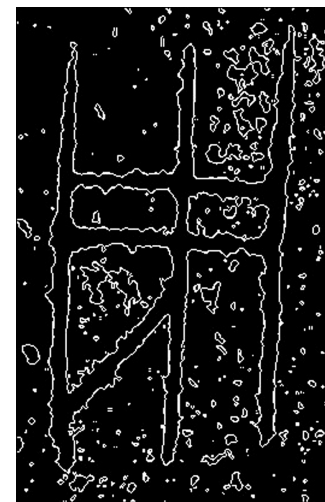

图 3 轮廓图

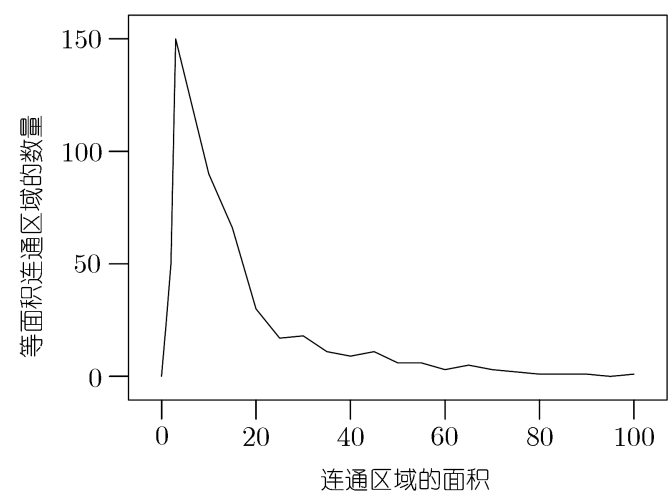

图 4 连通区域面积分布特征

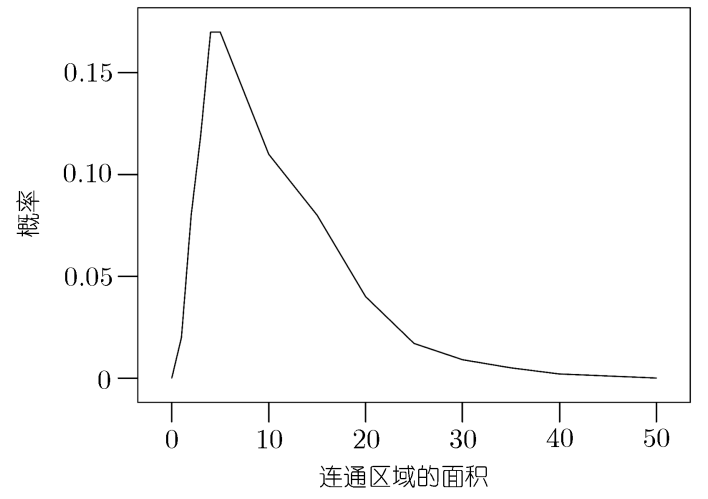

图 5 连通区域面积概率密度

其中 $\lambda>0, X$ 表示甲骨拓片上连通区域的面积, 则随机变量 $X$ 的数学期望为

$$
\mathrm{E}(X)=\sum_{i=1}^{\infty} i \frac{\lambda^{i}}{i !} \mathrm{e}^{-\lambda}=\lambda \mathrm{e}^{-\lambda} \sum_{i=1}^{\infty} i \frac{\lambda^{i-1}}{i !}=\lambda \mathrm{e}^{-\lambda} \sum_{i=1}^{\infty} \frac{\lambda^{i-1}}{(i-1) !} .
$$

令 $k=i-1$, 则

$$
\lambda \mathrm{e}^{-\lambda} \sum_{i=1}^{\infty} \frac{\lambda^{i-1}}{(i-1) !}=\lambda \mathrm{e}^{-\lambda} \sum_{i=1}^{\infty} \frac{\lambda^{k}}{k !}=\lambda \mathrm{e}^{-\lambda} \mathrm{e}^{\lambda}=\lambda .
$$

以 320 pixel $\times 512$ pixel 的图像为例, 根据实验数据, 连通区域的面积与连通区域数目的关系如图 4 所示. 从图 4 中可以看出, 噪声区域的面积通常在 50 个像素以下, 字形笔划区域的面积一般在 100 个像素以上. 通过计算, 我们得出甲骨拓片连通区域面积分布的概率密度函数, 可以表示如下:

$$
P_{i}=P\{X=i\}=\frac{5^{i}}{i !} \quad(i=0,1,2, \ldots),
$$

甲骨拓片连通区域面积的数学期望为 5.0. 图 5 是甲骨拓片连通区域的概率密度图.

\section{2 泊松分布的噪声区域填充方法}

以 320 pixel $\times 512$ pixel 的图像为例, 根据甲骨拓片上连通区域面积的分布特征, 基于泊松分布的 甲骨拓片图像区域填充算法如下: 

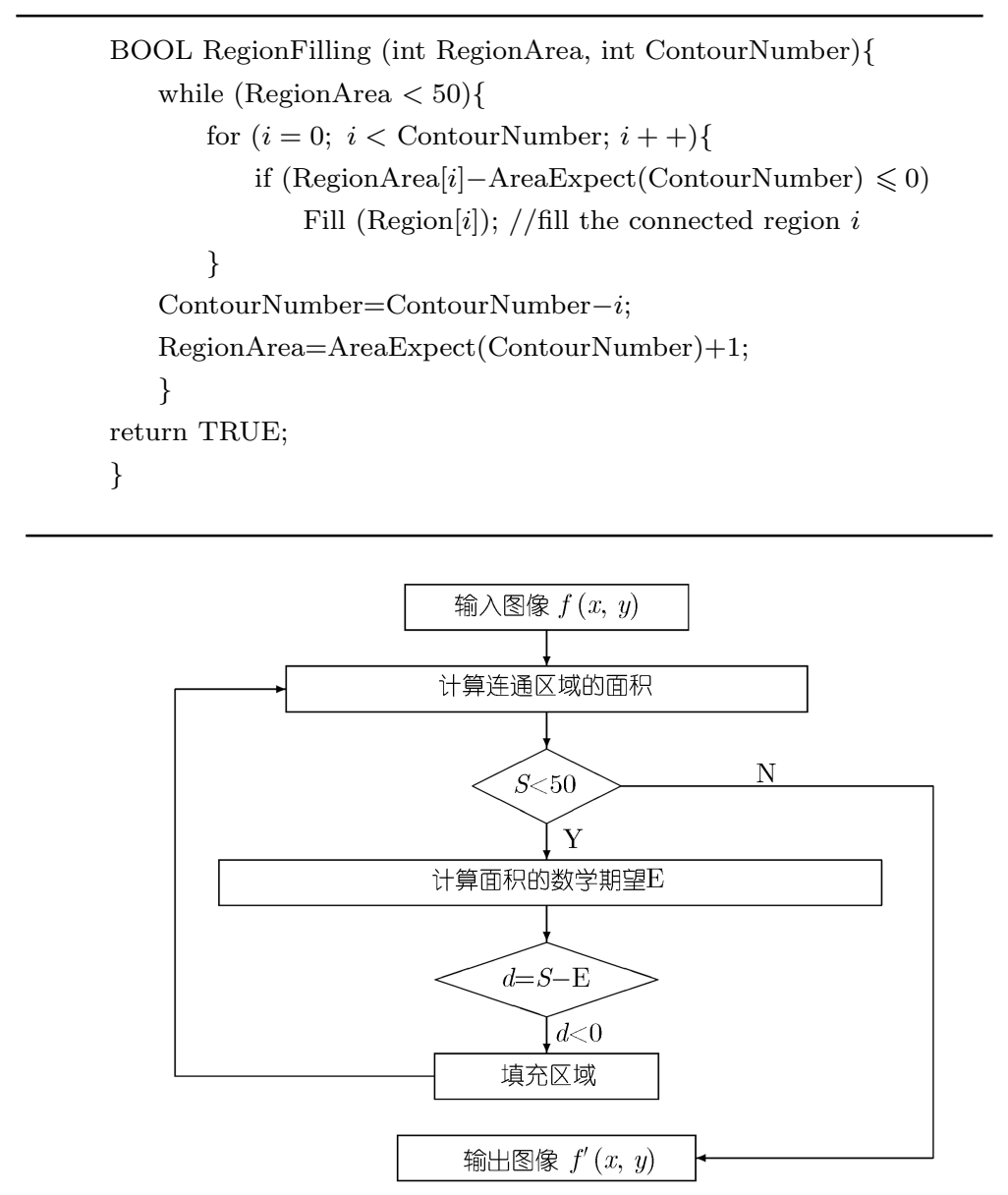

图 6 算法流程图

上述算法中, RegionArea [i] 表示序号为 $i$ 的连通区域的面积, Region $[i]$ 表示序号为 $i$ 的连通区域, Fill(Region $[i])$ 表示对序号为 $i$ 的连通区域进行填充. ContourNumber 表示甲骨拓片上连通区域的数 目, AreaExpect(ContourNu-mber) 表示对 ContourNumber 个连通区域的面积计算数学期望. 当甲骨 拓片上连通区域的面积大于 50 时, 退出循环, 输出图像. 算法步骤如下:

步骤 1 计算甲骨拓片上连通区域的面积.

步骤 2 如果出现面积小于 50 像素的连通区域, 计算连通区域面积的数学期望, 否则输出图像 并退出.

步骤 3 如果连通区域的面积小于数学期望, 则填充这些区域, 并继续执行步骤 2 .

算法流程可以表示如图 6.

\section{3 实验结果}

通过计算连通区域面积 $S$ 与区域面积的数学期望 $\mathrm{E}$ 的差值 $d=S-\mathrm{E}$, 当 $d<0$ 时, 将面积为 $S$ 的连通区域识别为噪声区域, 并将之填充. 余下的连通区域面积同样服从泊松分布, 再按照上述步骤 进行计算. 循环上述步骤, 直到各连通区域的面积大于 50 时, 循环终止. 我们在 Apple Mac Pro 工作 


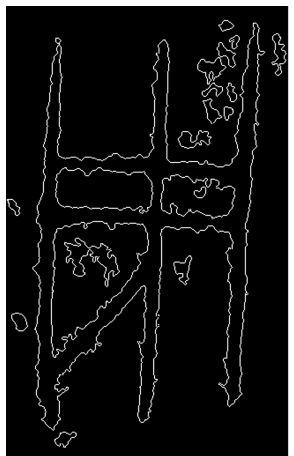

(a)

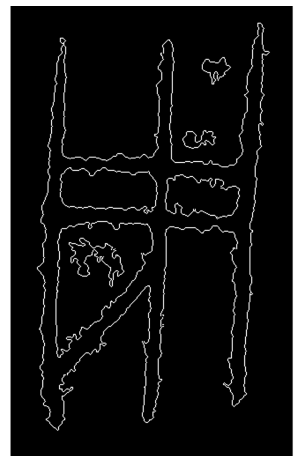

(b)

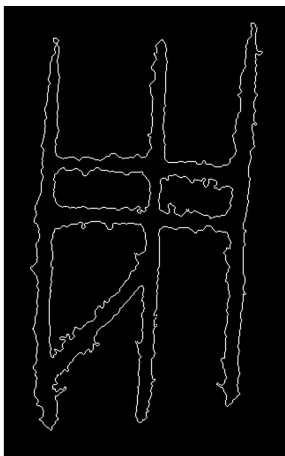

(c)

图 7 实验结果

站上在 Windows 环境下使用 Visual C++6.0 和 OpenCV1.1 实现了上述算法. 图 7(a) 为经过一次填 充操作后的图像, 图 7(b) 为经过二次填充操作后的图像, 图 7(c) 是经过基于泊松分布识别处理的甲 骨拓片图像区域填充效果.

\section{3 基于分形几何的字形图像复原方法}

\section{1 分形几何理论}

分形是 20 世纪 70 年代初由 Mandelbrot ${ }^{[14]}$ 引入的关于自相似性的一般概念, 是描述具有相似结 构的几何形状的工具, 后来发展成对各种复杂系统的自相似性进行研究的工具. 具有分形特征的表面 具有自相似性, $n$ 维欧氏空间中的有界集合 $A$, 若 $A$ 可以表示为其自身 $N_{r}$ 个互不覆盖的子集的并集 时, 则 $A$ 是自相似的. 此时, $A$ 的分形相似维数定义为

$$
D=\frac{\lg \left(N_{r}\right)}{\lg (1 / r)},
$$

其中, $r$ 是所有坐标方向上的尺度因子, $N_{r}$ 是有界集合 $A$ 的互不覆盖的子集个数. 对于图像而言, 把 二维图像视作一个三维空间中的一个表面 $(x, y, f(x, y))$, 其中 $f(x, y)$ 为图像 $(x, y)$ 位置处的灰度值, 于是图像灰度的变化情况将反映在该表面的粗粘程度上, 使用不同尺度去度量该表面, 得到的维数就 是图像的分形维数. 目前已有许多估计分形维数的方法, 例如频域方法 [15]、计盒维数法 ${ }^{[16]}$ 、数学形 态学的方法 ${ }^{[8]}$ 、差分盒维数算法 ${ }^{[17]}$ 等. 其中, 差分盒维数法由于易于计算、性能较好等优点, 在实际 中得到了广泛的应用.

差分盒维数法的主要思想是: 将 $m \times n$ 大小的图像分割成 $s \times s$ 的子块, $s$ 是介于 1 和 $m / 2$ 之间 的整数. 令 $r=s / m$, 将图像视为一个三维空间中的一个表面 $(x, y, f(x, y)),(x, y)$ 表示为像素点的平 面位置, 第三维用来表示像素点的灰度值 $f(x, y)$. 平面被分割成许多 $s \times s$ 的网格, 在每个网格上, 是 一列 $s \times s \times s$ 的盒子. 假设在图像的第 $(i, j)$ 个网格内图像灰度的最高值和最低值分别落在第 $l$ 个和 第 $k$ 个盒子内, 则 $N_{r}$ 在第 $(i, j)$ 个网格内的分布 $n_{r}(i, j)$ 为 $n_{r}(i, j)=l-k+1 . n_{r}(i, j)$ 是覆盖第 $(i, j)$ 网格中的图像所需的盒子数, 则覆盖整个图像所需的盒子数 $N_{r}$ 为

$$
N_{r}=\sum_{i, j} n_{r}(i, j)
$$




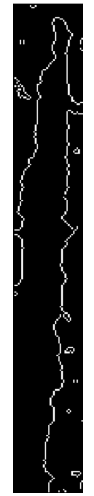

图 8 图 3 之局部放大

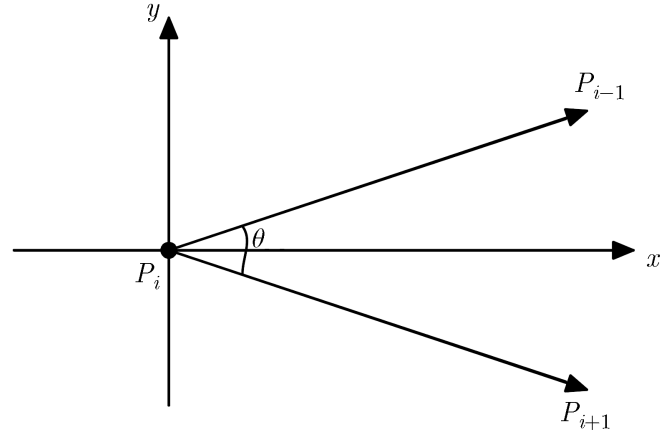

图 9 特征点的提取

对于不同的 $r$, 可以得到不同的 $N_{r}$ 值, 采用最小二乘法可以拟合出 $\lg N_{r} \sim \lg (1 / r)$ 的斜率, 即可得到 对应的分形维数值 $D$.

\section{2 甲骨拓片字形图像边缘的分形特征}

甲骨拓片在地下深埋上千年, 由于受到腐蚀, 字形边缘呈现出连绵起伏的锯齿形状. 甲骨拓片字 形图像的锯齿形状边缘部分与整体具有相似性, 具备分形特征, 如图 8 所示. 甲骨文字形是刻写在坚 硬的材料上, 大部分字形笔划具有锋利的端点. 这些端点指示了字形笔划的位置, 是甲骨文字形的重 要信息. 这些端点可以通过一条笔划相邻的两条边缘夹角 $\theta$ 计算出来. 夹角 $\theta$ 可以计算如下:

$$
\theta=\arccos \left(\frac{V_{1} \cdot V_{2}}{\left|V_{1}\right|\left|V_{2}\right|}\right) \quad \theta \leqslant \pi,
$$

其中, $V_{1}$ 和 $V_{2}$ 分别是两条曲线的公共端点和它们的另一端点所形成的向量 $P_{i} \boldsymbol{P}_{i-1}$ 和 $P_{i} \boldsymbol{P}_{i+1}$. 设 $M$ 是夹角 $\theta$ 的阈值, 如果 $\theta \leqslant M$, 系统就认为点 $P_{i}$ 是笔划的端点. 采用这种方式可以把所有的端点都提 取出来, 提取端点的这种方法如图 9 所示.

构成甲骨文字形的笔划复杂, 并且数量巨大, 但是组成笔划的笔段却是简单的和有限的. 可以通 过平滑构成笔划的笔段来达到平滑字形笔划边缘的效果. 甲骨文字形的笔划可以归结为由 19 种不同 的笔段组成 ${ }^{[18]}$. 通过提取特征点, 可以得到所有的笔段, 并分别对这些笔段进行压缩变换.

在平面欧氏空间中, 伸缩变换可以表示为

$$
\left(\begin{array}{l}
x^{\prime} \\
y^{\prime}
\end{array}\right)=\left(\begin{array}{ll}
r & 0 \\
0 & r
\end{array}\right)\left(\begin{array}{l}
x \\
y
\end{array}\right),
$$

上式中, $r$ 为伸缩比, $r$ 大于 1 时为伸长变换, $r$ 小于 1 时为压缩变换. 在对字形轮廓上的特征点进行 压缩变换操作时, 为了既对字形边缘进行平滑, 同时又保持甲骨文字形笔划的基本走向和基本形状, 对 特征点的坐标与其相邻点的坐标进行加权处理, 可以用矩阵表示如下:

$$
\boldsymbol{x}^{\prime}=\left(\begin{array}{lllll}
x_{i+2} & x_{i+1} & x_{i} & x_{i-1} & x_{i-2}
\end{array}\right)\left(\begin{array}{c}
w_{i+2} \\
w_{i+1} \\
w_{i} \\
w_{i-1} \\
w_{i-2}
\end{array}\right),
$$




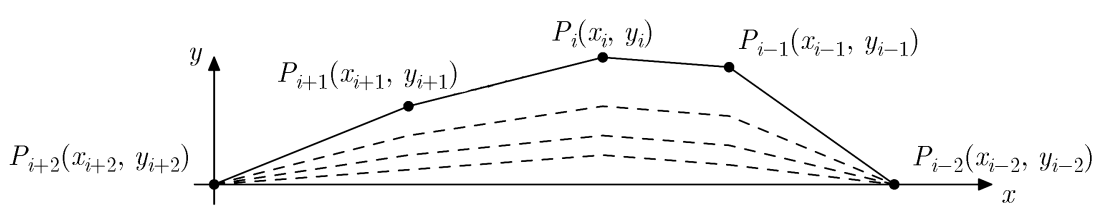

图 10 字形边缘平滑示意图

或者

$$
\boldsymbol{y}^{\prime}=\left(\begin{array}{lllll}
y_{i+2} & y_{i+1} & y_{i} & y_{i-1} & y_{i-2}
\end{array}\right)\left(\begin{array}{c}
w_{i+2} \\
w_{i+1} \\
w_{i} \\
w_{i-1} \\
w_{i-2}
\end{array}\right), \quad i \geqslant 2 .
$$

字形轮廓边缘平滑操作如图 10 所示. $P_{i+2}\left(x_{i+2}, y_{i+2}\right) 、 P_{i+1}\left(x_{i+1}, y_{i+1}\right) 、 P_{i-1}\left(x_{i-1}, y_{i-1}\right) 、 P_{i-2}\left(x_{i-2}\right.$, $\left.y_{i-2}\right)$ 分别是 $P_{i}\left(x_{i}, y_{i}\right)$ 相邻的 4 个点, 以直线 $P_{i+2} P_{i-2}$ 建立 $x$ 轴, 图 10 中虚线是经过加权处理压缩 变换后的图形. 从图 10 可以看出, 经过加权处理后, 字形边缘变得平滑, 同时又保持了字形笔划的基 本走向和形状.

经过统计, 甲骨拓片字形图像边缘的分形维数在 1.2-1.3 之间, 设定分形维数阈值 $T$, 当压缩变换 后的图像分形维数小于 $T$ 时, 处理结束. 直线的分形维数是 1 , 通常, 甲骨拓片字形图像分形维数阈值 设为 1.05 即可. 算法步骤如下:

步骤 1 使用 freeman 链码对输入图像 $f(x, y)$ 进行轮廓跟踪.

步骤 2 在 $x$ 或者 $y$ 方向上对 $f(x, y)$ 进行压缩变换, 得到图像 $f^{\prime}(x, y)$.

步骤 3 计算图像 $f^{\prime}(x, y)$ 的分形维数 $D$, 如果 $D<T$ 则输出图像, 否则转步骤 2 .

算法流程图如图 11 所示.

\section{3 实验结果}

我们在 Apple MacPro 工作站上在 Windows 环境下, 用 Visual C ++6.0 和 OpenCV1.1 实现了上 述算法. 图 12, 13 是分形维数阈值分别为 $1.2,1.1,1.05$ 时经过压缩变换后的输出的图像. 图像经过压 缩变换后, 图像边缘的锯齿状态得到了平滑. 图 14 是对甲骨拓片字形边缘进行数据拟合的结果, 字形 边缘得到了轻度的平滑, 但是仍然非常粗糙, 效果不如图 12, 13. 表 1 给出了压缩变换和数据拟合的 时间花费.

\section{4 结论}

甲骨文是我国迄今发现的最早的一种成熟文字系统, 如何有效地对甲骨文进行展示和保护具有重 要的意义. 本文分析了甲骨拓片上噪声以及字形图像边缘的特点, 通过计算甲骨拓片上连通区域面积 的分布特征, 提出了一种基于泊松分布和区域填充的图像去噪方法. 根据各连通区域的面积与数学期 望的差值, 所有的连通区域都被识别处理. 当差值小于数学期望时, 连通区域被识别为噪声区域并被 填充, 从而笔划区域被保留. 实验结果显示, 该方案可以有效地去除甲骨拓片上的噪声区域. 本文分析 


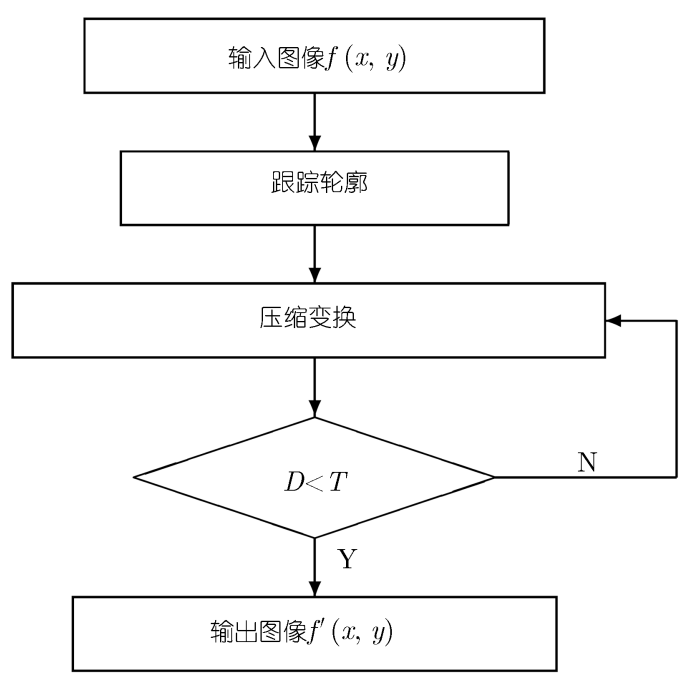

图 11 算法流程图

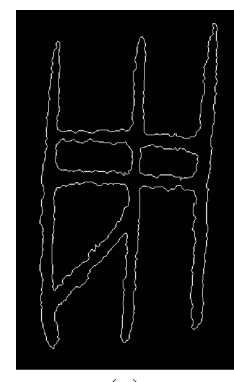

(a)

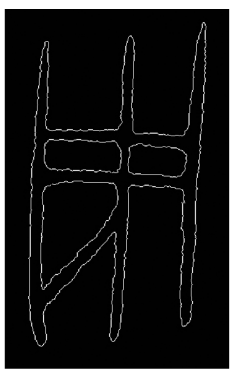

(b)

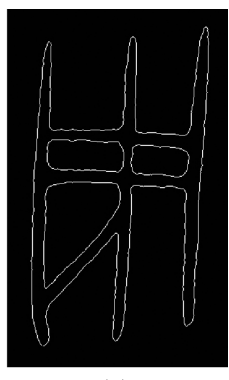

(c)

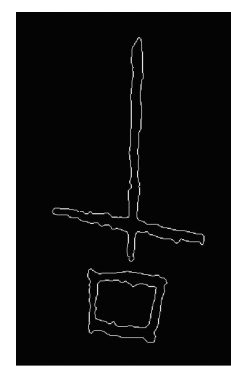

(a)

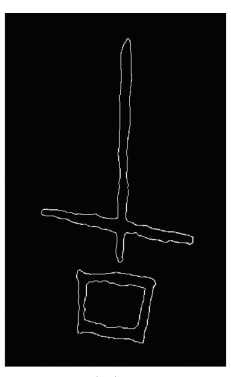

(b)

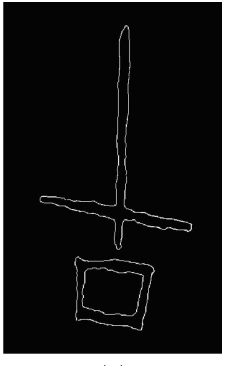

(c)

图 12 实验结果

图 13 实验结果

表 1 压缩变换和数据拟合的时间花费

\begin{tabular}{ccc}
\hline 算法 & 时间消耗 $(\mathrm{s})$ & 实验结果 \\
\hline 压缩变换 & 0.51 (图 12), $0.45($ 图 13) & 平滑效果较好 \\
数据拟合 & $0.16($ 图 $14(\mathrm{a})), 0.15($ 图 14(b)) & 轻度平滑, 仍然粗䊁 \\
\hline
\end{tabular}

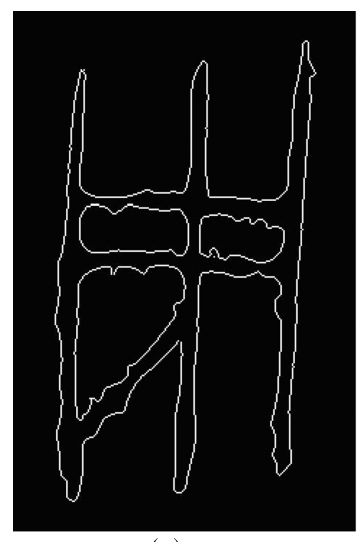

(a)

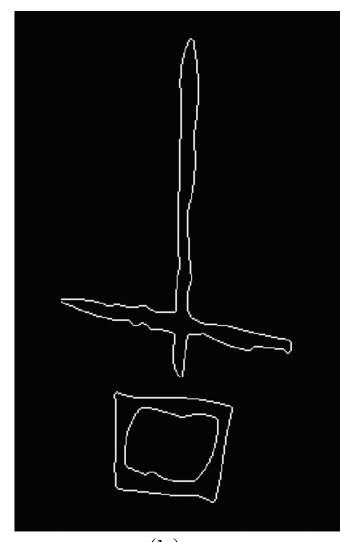

(b)

图 14 数据拟合实验结果 
了甲骨拓片上字形边缘的分形特征, 提出了基于分形几何的字形复原方法. 通过计算字形边缘的分形 维数, 对甲骨拓片上字形的笔划和笔段进行加权的压缩变换, 从而对甲骨拓片字形的边缘进行平滑. 最 后, 可以获得接近甲骨文字形原貌的满意字形. 实验结果显示, 该方案可以有效地对甲骨拓片字形边 缘进行平滑, 并保持字形边缘的基本形状.

\title{
参考文献
}

1 Bertalmio M, Sapiro G, Caselles V, et al. Image inpainting. In: Proceedings of SIGGRAPH2000. Boston: AddisonWesley Professional, 2000. 417-424

2 Chan T, Shen J. Mathematical models for local deterministic inpaintings. SIAM J Appl Math, 2001, 62: 1019-1043

3 Chan T, Shen J. Non-texture inpainting by curvature-driven diffusions. J Visual Comm Image Rep, 2001, 12: 436-449

4 Oliveira M, Bowen B, McKenna R, et al. Fast digital image inpainting. In: Proceedings of IASTED Conference on Visualization, Imaging, and Image Processing. Marbella: ACTA Press, 2001. 261-266

5 Carr J C. Reconstruction and representation of 3D objects with radial basis functions. In: Proceedings of ACM SIGGRAPH2001. New York: ACM Press, 2001. 67-76

6 Esedoglu S, Shen J. Digital inpainting based on the Mumford-Shah-Euler image model. Eur J Appl Math, 2002, 13: $353-370$

7 Criminisi P P, Toyama K. Object removal by exemplar-based inpainting. In: Proceedings of IEEE Computer Society Conference on Computer Vision and Pattern Recognition. Washington: IEEE Computer Society, 2003. 721-728

8 Elad M, Starck J L, Querre P, et al. Simultaneous cartoon and texture image inpainting using morphological component analysis. Appl Comput Harmon Anal, 2005, 19: 340-358

9 Bertozzi E S, Gillette A. Inpainting of binary images using the Cahn-Hilliard equation. IEEE Trans Image Process, 2007, 16: 285-291

10 Guerrero-Colón J A, Mancera L, Portilla J. Image restoration using space-variant Gaussian scale mixture in overcomplete pyramids. IEEE Trans Image Process, 2008, 17: 27-41

11 Henri P, Gosselin, Cord M. Active learning methods for interactive image retrieval. IEEE Trans Image Process, 2008, 17: $1200-1211$

12 Dobrosotskaya J, Andrea B L. A wavelet-laplace variational technique for image deconvolution and inpainting. IEEE Trans Image Process, 2008, 17: 657-663

13 Ma X H, Huang W F, Gu S T, et al. A method for converting Jiaguwen dot matrix to Jiaguwen outline font (in Chinese). Linguist Sci, 2004, 3: 3-11

14 Mandelbrot B B. Fractals: Form, Chance and Dimension. San Francisco: W. H. Freeman, 1977

15 Pentland A P. Fractal-based description of natural scenes. IEEE Trans Patt Anal Mach Intell, 1984, 6: 661-674

16 Chaudhuribb S N. Texture segmentation using fractal dimension. IEEE Trans Patt Anal Mach Intell, 1995, 17: 72-77

17 Chaudhuribb S N. An efficient differential box-counting approach to compute fractal dimension of image. IEEE Trans Syst Man Cybern, 1994, 24: 115-120

18 Ma X H, Fan X X, Yang Y M. An outline description based transform strategy for generating new style jiaguwen. J Comput Inf Syst, 2007, 3: 493-499

\section{Restoration method of characters on Jiagu rubbings based on Poisson distribution and fractal geometry}

\author{
GU ShaoTong ${ }^{1,2,3}$, FENG GeFei ${ }^{1,2,3}$, MA XiaoHu ${ }^{1,2,4}$ \& YANG YiMing ${ }^{1,2,3, *}$ \\ 1 Institute of Linguistics, Xuzhou Normal University, Xuzhou 221009, China; \\ 2 Jiangsu College Key Lab of Linguistic Sciences and Neuro-cognition Engineering, Xuzhou Normal University,
}


Xuzhou 221009, China;

3 Linguistic Science College, Xuzhou Normal University, Xuzhou 221009, China;

4 School of Computer Science and Technology, Soochow University, Suzhou 215006, China

*E-mail: yangym@xznu.edu.cn

\begin{abstract}
A scheme for character restoration of Jiagu rubbings based on Poisson distribution and fractal geometry is proposed in this paper. The distribution characteristics of the connected regions on Jiagu rubbings is analyzed, and all the connected regions are recognized according to differences between these and their mathematical expectations. Regions are recognized as noise regions and filled if the differences are negative, while stroke regions are reserved. The attributes of the character image edges on Jiagu rubbings are also analyzed. The fractal dimensions of the character edge of Jiagu rubbings are calculated by means of statistics. The feature points are obtained using the cosine of the angle between the two vectors of the point and the consecutive two points. Different compression transformations with weighted coordinates are performed on different strokes and segments of the characters so as to smoothen the character edge of Jiagu rubbings images. Experimental results show that our scheme could successfully clear noise regions and polish the character edge of Jiagu rubbings.
\end{abstract}

Keywords Jiagu rubbings, Poisson distribution, fractal geometry, fractal dimension, compression transformation, character restoration 